\title{
Enhancing Pretrained Models with Domain Knowledge
}

\author{
Yufei Feng*, Michael Greenspan and Xiaodan Zhu* \\ Electrical and Computer Engineering \& Ingenuity Labs Research Institute, Queen's University
}

\begin{abstract}
Unsupervised pretraining has recently achieved significant success on a wide variety of natural language processing tasks. An important problem that remains understudied is how to effectively adapt such models to specific domains, which is critical for many real-life applications. In this paper, we explore to enhance pretraining by leveraging two typical sources of domain knowledge: unstructured domain-specific text and structured (often human-curated) domain knowledge. We propose models to jointly utilize these different sources of knowledge, which achieve the state-of-the-art results on two tasks of different domains: stock price movement prediction and software bug duplication detection, by adapting publicly available pretrained models obtained on generic domain-free corpora like book corpora and news articles.
\end{abstract}

Keywords: Domain Adaptation, Dynamic Routing, Pretrained language Model

\section{Introduction}

Unsupervised pretraining represents one of the most recent advances in representation learning for natural language. The models have been shown to bring forward the state of the art of a wide range of NLP tasks by effectively leveraging large generic text corpora, e.g., news articles, Wikipedia, and book corpora [1,2], to learn domain-independent representation.

In many real-world natural language applications, tasks often fall into specific domains. More research is still needed to explore how to effectively adapt such models to specific domains-it is intriguing to develop models that can leverage both the advance of pretraining and abundant domain knowledge.

In this paper, we develop models to leverage two typical sources of domain knowledge: unstructured domain text and structured (often human-curated) domain knowledge. To utilize unstructured domain text, we find the weight adaptation strategy that adapts all pretrained parameters with domain corpora yields better results than the alternatives. Based on that, We build additional networks on top of the existing pretrained models. We proposed dynamic routing over sequence (DyROS) for the task. Motivated by the capsule network [3], we modify dynamic routing to leverage routingby-agreement for sequences, which iteratively adjusts routing parameters on-the-fly for different domains to learn domain-specific part-whole relationship and composition.

We further incorporate structured knowledge into DyROS. In addition, we also introduce different approaches to employing structured knowledge, including using such knowledge in typical components of a Transformer block, e.g., self-attention weights and value vectors [4].

Our best models achieve the state-of-the-art results on two tasks in very different domains: stock price movement prediction and software bug duplication detection, by adapting publicly available pretrained models obtained on generic, domain-free corpora like book corpora and news articles.

\section{Related Works}

Pretrained Models and Finetuning. Unsupervisedly trained models such as language models have long been used in many different NLP tasks. Among the recent advances, Dai and Le [5] showed that language models trained on a large unlabeled corpus improved many downstream tasks. The widely adopted ELMo [6] trains a language model recursively with a bi-directional recurrent neural network, and then treats the pretrained representations as features in other tasks but does not change task-specific model architectures. GPT [7] pretrained a model on a book corpus [8] using stacked

*feng.yufei@queensu.ca, xiaodan.zhu@queensu.ca 


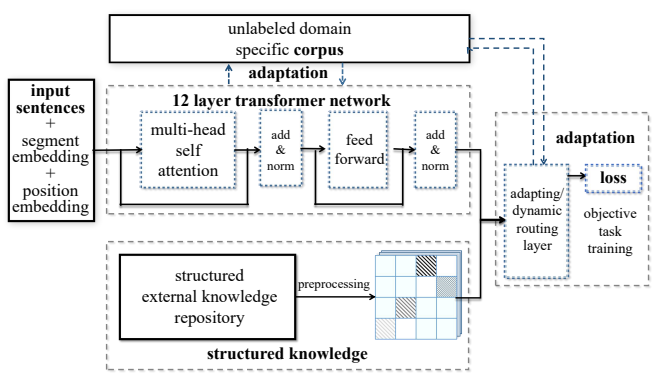

Figure 1. An overview of the model. Information from the upper block (Domain-adapted BERT Encoder) and the lower block (structured knowledge) are fed to adaptation layers of the model.

Transformer blocks [4] and transferred model architectures together with learned parameters to solve downstream tasks. BERT [2] is another state-of-the-art model built on Transformer.

Structured External Knowledge. Positing all information useful for target tasks resides in the training set is a strong assumption. Many recent neural-network-based models have started to incorporate external structured knowledge. Chen, Zhu, Ling, and Inkpen [9] showed that lexical relationship from WordNet [10] greatly improved model performance in natural language inference (NLI) tasks. Recent works also proposed models that involve inference over a graph. Socher, Chen, Manning, and $\mathrm{Ng}$ [11] proposed a neural tensor network, which uses a bi-linear operator to predict pair-wise relation in the knowledge base. Schlichtkrull, Kipf, Bloem, Van Den Berg, Titov, and Welling [12] proposed a Graph Convolutional Network that reasons over graph structure.

Dynamic Routing and Attention. Sabour, Frosst, and Hinton [3] first proposed the capsule network with a dynamic routing algorithm, and Hinton, Sabour, and Frosst [13] further perform dynamic routing with mixture models. Unlike traditional neural networks, where the output layer is a fixed projection of the input representation, a capsule network first generates votes from the lower-level representations and finds dominating components (votes that agree) through dynamic weights adjustment. Dynamic routing was first proposed to support coincidence filtering through agreement and hence better model part-whole relationship.

\section{The Models}

Figure 1 shows a high-level overview of our framework. The input is composed of word tokens of input sentences, their positional encoding, and the segment labels used in Transformer-based models (see [4] for details). They are fed into pretrained sentence encoder learned on generic corpora. Unstructured domain text will then be used for adaptation, and we propose models to incorporate structured knowledge, taking the form of triples $\left(w_{i}, w_{j}, r\right)$, where $w_{i}, w_{j}$ are items and $r$ is the relation.

Adapted BERT Encoder. Pretrained language models such as BERT [2] and GPT [1] have shown to be capable of effectively leveraging large domain-free corpora such as book corpora [8] and Wikipedia text. It is however unlikely that the models can capture domain semantics and knowledge that do not exist in the pretraining data. Our adaptation scheme follows BERT pretraining [2]. We organized unlabeled sentences into pairs, and randomly mask or substitute some of the words in each pair. The research of [14-16] presents a thorough comparison of different weight transfer methods. In our case the final evaluation is not the perplexity of the adapted language model, but its impact on downstream tasks.

Dynamic Routing Over Sequence (DyROS). We propose dynamic routing over sequence (DyROS), motivated by the Capsule Network [3]. We adapt dynamic routing for sequences to leverage 
its good property: the routing-by-agreement can iteratively adjust the routing parameters on-the-fly, depending on the input data. We expect it to help capture domain-specific (part-whole) agreement and composition.

Capsule networks $[3,13]$ compute votes from lower-level capsules/vectors to high-level ones. In the original model the output capsules/vectors are not ordered. In this work, we adapt the model to track word positions in order to model sentences.

Given an input sequence $\mathbf{X}=\left[x_{1}, x_{2}, \ldots, x_{n}\right]$ with $x_{i}$ being the word embedding of the $i^{t h}$ token, let $H$ denote the output of adapted BERT encoder discussed above:

$$
H=\left[h_{1}, h_{2}, \ldots h_{n}\right]=B E R T(\mathbf{X})
$$

Then DyROS feeds the obtained $H$ into its voting layer. Same as in the capsule network detailed in [3], for a lower-level capsule/vector $h_{i}$ derived above in Equation 3.1 and a higher-level capsule $h_{j}^{\prime}$ (the vector at position $j$ in the output sequence), we need to first compute the vote, $\hat{v}_{j \mid i}$, i.e., intuitively 'what $h_{i}$ thinks the voting target $h_{j}^{\prime}$ should look like'.

More specifically, to compute $\hat{v}_{j \mid i}$, unlike in [3], we want $h_{j}^{\prime}$ to not only depend on $h_{i}$ but also to track the low-level capsule at position $j$ (i.e., $h_{j}$ ). This strategy is widely used in many existing sequence models, e.g., Transformer, through their self-attention mechanism.

We propose to calculate $\hat{v}_{j \mid i}$ with the following formula:

$$
\begin{gathered}
\hat{v}_{j \mid i}=f\left(h_{j}, h_{i}\right) \\
=\operatorname{ReLU}\left(W *\left[\phi\left(h_{j}\right), \psi\left(h_{i}\right)\right]\right) \\
\phi\left(h_{j}\right)=\operatorname{Re} L U\left(W_{\phi} * h_{j}\right), \quad \psi\left(h_{i}\right)=\operatorname{ReLU}\left(W_{\psi} * h_{i}\right)
\end{gathered}
$$

where, $\phi$ and $\psi$ are two independent transformations, and $W$ transforms the concatenated features into the votes. The use of $\phi$ and $\psi$ are motivated by the query and key in Transformer.

Unlike in capsule network [3], where each $\langle i, j\rangle$ pair has its own weight matrix, we share weights here; that is, we only have one $W_{\phi}$ in each layer, same for $W_{\psi}$ and $W$, which is motivated by [17]. This significantly reduces the number of parameters and increase training efficiency, while the original capsule network and dynamic routing are known to be very slow.

The high-level capsules now can be computed from the votes based on dynamic routing:

$$
h_{j}^{\prime}=\operatorname{squash}\left(\sum_{i} c_{i j} * \hat{v}_{j \mid i}\right)
$$

where squash is the normalization function defined in [3] and $c_{i j}$ is the routing parameters.

Since $h_{j}^{\prime}$ is the agreed majority of $\hat{v}_{j \mid i}$, and it is computed on-the-fly depending on input (here, different domain data), we expect it to capture domain-specific composition. We use the dynamic routing algorithm described in [3] (specified as Algorithm/Procedure 1) to perform routing.

DyROS with Structured Knowledge (DyROS-Kn). In this paper, we use domain knowledge of concepts and relations_-given a pair of words $w_{i}, w_{j}$ together with $d$ different relations, we have a $d$-dimensional vector $e_{i j}$.

$$
\text { Knowledge }_{d}\left(w_{i}, w_{j}\right)=e_{i j}(d)=\mathbf{K n}(i, j, d)
$$

Structured domain knowledge $e_{i j}$ can be treated as an extra feature in the voting process of DyROS. Intuitively, structure knowledge between the word $w_{i}$ and $w_{j}$ can be used to help capture semantic relationship between $h_{j}$ and $h_{i}$ above in Equation 3.1.

To incorporate knowledge triple $e_{i j}$ into KyROS, we modify Equation 3.3 as follows:

$$
\begin{aligned}
\hat{v}_{j \mid i} & =f\left(h_{j}, h_{i}, e_{i j}\right) \\
& =\operatorname{Re} L U\left(W *\left[\phi\left(h_{j}\right), \psi\left(h_{i}\right), e_{i j}\right]\right)
\end{aligned}
$$


Table 1. Examples from the bug duplication detection dataset and the stock price movement prediction dataset. A bolded word in brackets indicated the true label of the sample, and we only show part of the news sample due to limited space.

\begin{tabular}{|c|l|}
\hline Debug Data & Content \\
\hline Sentence 1 & Add possibility to manage available search engines from preferences and sidebar \\
Sentence 2 & Advanced add/remove engine functions for search sidebar (Duplicate) \\
\hline Stock Data & Content \\
\hline Title & Ford to invest \$773 million across southeast Michigan \\
Abstract & Ford Motor Co ( F.N ) plans to invest ... and capacity expansions ... (Up). \\
\hline
\end{tabular}

\section{Data}

To evaluate the models, we use two real-world domain-specific datasets, each is influential benchmark data in their domains and these two domains are very different from each other.

Software Bug-Description Duplication Detection. Given a pair of sentences describing something wrong in the software testing process, the goal is to predict whether those two sentences are generated by different engineers when reviewing the same module. An example of duplicated bugs can be found in the upper half of Table 1. The dataset contains 66,484 labeled sentence pairs, and we use the same division as in [18] for a fair comparison. We also collected from the raw data repository provided in [18] a reasonable amount of unlabeled sentences for language model adaptation.

We extract domain knowledge from Mozilla Developer Documentation ${ }^{1}$ term glossary, which contains 440 technical terms (topics). For each topic, we collect its name, one sentence description, content page text, and text from external links referenced by the content page. We only consider external links within the documentation or Wikipedia. For an arbitrary pair of words A and B, five relation scores are defined as follows:

- Lexical Same: A and B are the same word.

- Lexical Contain: A is a sub-word of B, and vice versa. This situation is likely to happen when word B is divided into pieces during BERT word-piece encoding.

- Documentation Contain: A is one of the 440 terms or appears in compact term descriptions, and B is in the content page of that term, vice versa.

- Close Relation: A and B appear in the content page of the same glossary term.

- General Relation: A and B appear in the content and external link page of the same term.

The first two types of relations are defined by binary categories, while others are defined through the probability of occurrence. The score of Documentation Contain and Close/General Relation is:

$$
\begin{array}{r}
P_{\text {contain }}=\max \left(P\left(B_{\text {content }} \mid A_{\text {title }}\right),\right. \\
\left.P\left(A_{\text {content }} \mid B_{\text {title }}\right)\right) \\
P_{\text {related }}=\sqrt{P(A \mid B) * P(B \mid A)}
\end{array}
$$

Stock Price Movement Prediction with Finance News. Stock price movement prediction has recently been explored in $[19,20]$. However, the data used in those previous works is unsatisfactory either in size or data quality. Following [19], we propose a new dataset based on existing sourcesthe dump of Reuters Stock Market News from 12/20/2006 to 12/31/2013. The goal is to predict individual company stock price movement around news releasing dates.

We obtained 16,812 samples which we then split into training, validation and test set of 10,812, 1,000 and 5,000 samples respectively. The data is arranged and split in regular time order. We also obtained approximately 1.25 million sentences for adaptation.

\footnotetext{
${ }^{1}$ https://developer.mozilla.org/en-US
} 
Table 2. Overall performance of different models, measured in test accuracy. The adaptation stage and the structured domain knowledge affects the performance incrementally.

\begin{tabular}{|l|c|c|}
\hline Models & Stock & Debug \\
\hline Previous SOTA & $54.69 \%$ & $92.66 \%$ \\
BERT & $54.82 \%$ & $97.12 \%$ \\
BERT+DyROS.Adp & $\mathbf{5 6 . 0 2} \%$ & $\mathbf{9 7 . 4 8} \%$ \\
BERT+DyROS.Adp+Kn & $\mathbf{5 6 . 4 6} \%$ & $\mathbf{9 7 . 6 0} \%$ \\
\hline
\end{tabular}

We use Sentiment WordNet Lexicon database [21] to enrich the word level features. Simultaneously, we build bags of keywords that best describe the stock movement or company situation. Example words in these groups include 'high', 'loss', 'plummet', 'resign' etc. Those knowledge are stored as relations between the classification token [CLS] and the corresponding word.

\section{Experiments}

We first adapt the pretrained model on the unlabeled corpus. In our experiment, we keep our adaptation hyper-parameters to be same as BERT pretraining. In each epoch, we randomly generate different mask locations and sentence combinations, while limiting the maximum number of masked positions to 10 for each sentence pair. We use parameters learned in the adaptation stage to initialize the fine-tuning network, where the structured domain knowledge is incorporated.

We present the test accuracy of both tasks in Table 2. Due to the stochastic nature of the financial market, the BERT baseline model performs slightly better than a random guess on the stock movement prediction task, and this is similar to the case in $[19,20]$. The baseline model is capable of reproducing the state-of-the-art performance in [19], and we report its test accuracy the on much larger dataset in Table 2. For the software bug duplication detection task, the BERT baseline we used already outperforms the previous state-of-the-art[18] by a large margin.

Our models further improve over the BERT baselines consistently, showing the benefit from two the sources: the adaptation that utilizing unstructured domain text and the modeling of structured knowledge. Comparison of test accuracies on BERT and adapted BERT shows that directly adapting all pretrained parameters yields significant improvement, and our best results are achieved by leveraging two sources of domain knowledge with the dynamic routing method proposed (DyROS-Kn), whose parameters are trained simultaneously during adaptation stage.

\section{Conclusions}

Pretrained models like BERT has achieved a considerable success in learning representation for natural language, improving the state of the art on a wide range of NLP tasks. In this paper, we explore to adapt pretrained models with unstructured domain corpora and structured domain knowledge. The models we propose can effectively leverage both types of domain knowledge to achieve the state-of-the-art performance on two tasks in very different domains: stock price movement prediction and software bug duplication detection, by adapting publicly-available pretrained models obtained on generic domain-free corpora like book corpora and news articles. In addition, we provide a detailed comparison on different adaptation strategies and various approaches of incorporating structured knowledge.

\section{Acknowledgement}

This research is supported by NSERC Discovery Grant, Accelerator Supplement and DND Supplement. 


\section{References}

[1] A. Radford, J. Wu, R. Child, D. Luan, D. Amodei, and I. Sutskever. "Language Models are Unsupervised Multitask Learners". In: (2019).

[2] J. Devlin, M.-W. Chang, K. Lee, and K. Toutanova. "BERT: Pre-training of Deep Bidirectional Transformers for Language Understanding”. In: arXiv preprint arXiv:1810.04805 (2018).

[3] S. Sabour, N. Frosst, and G. E. Hinton. "Dynamic routing between capsules". In: Advances in neural information processing systems. 2017, pp. 3856-3866.

[4] A. Vaswani, N. Shazeer, N. Parmar, J. Uszkoreit, L. Jones, A. N. Gomez, Ł. Kaiser, and I. Polosukhin. “Attention is all you need". In: NeurIPS. 2017, pp. 5998-6008.

[5] A. M. Dai and Q. V. Le. "Semi-supervised sequence learning". In: Advances in neural information processing systems. 2015, pp. 3079-3087.

[6] M. E. Peters, M. Neumann, M. Iyyer, M. Gardner, C. Clark, K. Lee, and L. Zettlemoyer. "Deep contextualized word representations". In: Proc. of NAACL. 2018.

[7] A. Radford, K. Narasimhan, T. Salimans, and I. Sutskever. "Improving language understanding by generative pre-training". In: (2018).

[8] Y. Zhu, R. Kiros, R. Zemel, R. Salakhutdinov, R. Urtasun, A. Torralba, and S. Fidler. "Aligning books and movies: Towards story-like visual explanations by watching movies and reading books". In: Proceedings of the IEEE international conference on computer vision. 2015, pp. 19-27.

[9] Q. Chen, X. Zhu, Z.-H. Ling, and D. Inkpen. "Natural language inference with external knowledge". In: (2018).

[10] G. A. Miller. "WordNet: a lexical database for English". In: Communications of the ACM 38.11 (1995), pp. 39-41.

[11] R. Socher, D. Chen, C. D. Manning, and A. Ng. "Reasoning with neural tensor networks for knowledge base completion". In: Advances in neural information processing systems. 2013, pp. 926-934.

[12] M. Schlichtkrull, T. N. Kipf, P. Bloem, R. Van Den Berg, I. Titov, and M. Welling. "Modeling relational data with graph convolutional networks". In: European Semantic Web Conference. Springer. 2018, pp. 593-607.

[13] G. E. Hinton, S. Sabour, and N. Frosst. "Matrix capsules with EM routing”. In: (2018).

[14] M. Ma, M. Nirschl, F. Biadsy, and S. Kumar. "Approaches for Neural-Network Language Model Adaptation.”In: INTERSPEECH. 2017, pp. 259-263.

[15] L. Mou, Z. Meng, R. Yan, G. Li, Y. Xu, L. Zhang, and Z. Jin. "How transferable are neural networks in nlp applications?" In: arXiv preprint arXiv:1603.06111 (2016).

[16] J. Howard and S. Ruder. "Universal language model fine-tuning for text classification". In: arXiv preprint arXiv:1801.06146 (2018).

[17] D. Yoon, D. Lee, and S. Lee. "Dynamic self-attention: Computing attention over words dynamically for sentence embedding". In: arXiv preprint arXiv:1808.07383 (2018).

[18] K. Aggarwal, F. Timbers, T. Rutgers, A. Hindle, E. Stroulia, and R. Greiner. "Detecting duplicate bug reports with software engineering domain knowledge". In: Journal of Software: Evolution and Process 29.3 (2017), e1821.

[19] X. Ding, Y. Zhang, T. Liu, and J. Duan. "Using structured events to predict stock price movement: An empirical investigation". In: Proceedings of the 2014 Conference on Empirical Methods in Natural Language Processing (EMNLP). 2014, pp. 1415-1425.

[20] Y. Xu and S. B. Cohen. "Stock Movement Prediction from Tweets and Historical Prices". In: Proceedings of the 56th Annual Meeting of the Association for Computational Linguistics (Volume 1: Long Papers). Melbourne, Australia: Association for Computational Linguistics, 2018, pp. 1970-1979. URL: http://aclweb.org/anthology/P18-1183.

[21] S. Baccianella, A. Esuli, and F. Sebastiani. "Sentiwordnet 3.0: an enhanced lexical resource for sentiment analysis and opinion mining.” In: Lrec. Vol. 10. 2010. 2010, pp. 2200-2204. 